\title{
Defining Academia Influences on Mobility, Identity, and Culture of Deaf Scholars in Higher Education
}

Trisha Houston, Ed.D.

California State University, Fresno 
What made me curious? 


\section{Background of the Study}

- Growth of Deaf Scholars ${ }^{1}$

- Lived experiences of Deaf academics being limited in research

1 (Garberoglio, Cawthon, \& Sales, 2017) 


\section{- Perceived Fear Factors in Academia $^{2}$}

\section{•"If I hire you ... I have to ..."}




\section{Most Prominent Theories used:}

\section{Social Phenomenology ${ }^{3}$ Deaf Critical Theory ${ }^{4}$ Deaf Epistemology ${ }^{5}$}

3 (Moustakas, 1994), 4 (Gertz, 2003), 5 (Hauser et al., 2010) 


\section{Exclusivity of Deaf faculty in data}

-ASL users

-Works in college/universities 


\section{Mixed Method}

\section{(Timeline)}

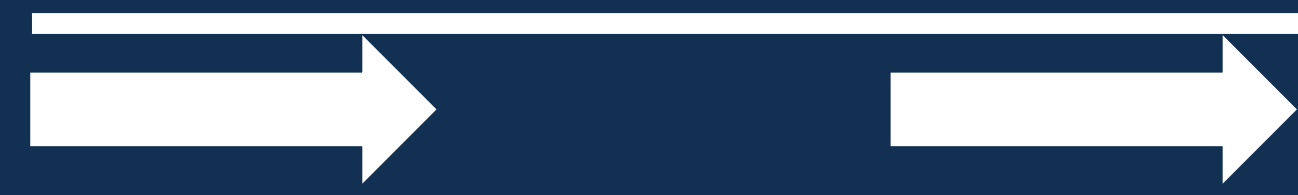

Start

Finish

December 2017

March 2018 


\section{Correlations}

\section{The desire to teach increased when barriers decreased.$$
(r=-.44, p=.008)^{*}
$$ 


\section{Personal Successes \& Barriers}

Positive Results

Academic mobility supported

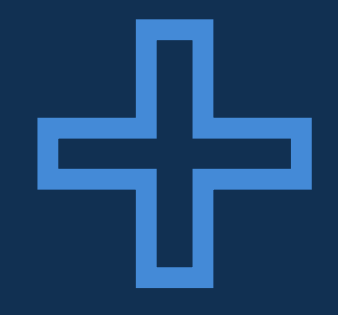

\section{Negative Results}

Constant fight for access 
Out of 55 respondents, $47 \%$ reported that they have experienced marginalization from other Deaf faculty. 


\section{Qualitative Results}

-Two sources of data Surveys and Interviews

-Thematic Coding and Triangulation 
Cultural competence

- Accessibility - Audism

- Burden (Academic and

Administration)

- "Good Enough"

- Proxy

Economic Issues 


\section{Elitism}

"You are not deaf enough"

\section{"Told me I needed to take Deafhood workshops to decolonize myself."}




\section{Collaboration}

\section{"Having a Deaf-heart"}

"Working with hearing people who truly believed Deaf people were equal." 


\section{Discussion}

Concerns about accessibility and the need of qualified interpreters for academic level (quality) 
-Cultural competence 6 training for all levels

-Role of Human Resources Departments in all Higher Education Institutions 


\section{Mentoring and Partnerships}

Communicating and imparting knowledge through sharing 
The power of Proxy is something we might overlook.

\section{We should grab the opportunity to work with others.}

